\title{
SPEAKER ADAPTATION USING MAXIMUM LIKELIHOOD GENERAL REGRESSION
}

\author{
Mohamad Hasan Bahari, Hugo Van hamme
Center for Processing Speech and Images (PSI), Department of Electrical Engineering (ESAT), KU Leuven, Belgium
MohamadHasan.Bahari@esat.kuleuven.be, hugo.vanhamme@esat.kuleuven.be

\begin{abstract}
In this paper, a new method called Maximum Likelihood General Regression (MLGR) is introduced for speaker adaptation. Gaussian means of a speaker independent (SI) model are adapted to the data of a new speaker by assuming a non-linear mapping from the SI Gaussian means to the adapted Gaussian means. MLGR performs a non-linear regression between ML estimates of the means and the SI means using General Regression Neural Network. The proposed method is evaluated on the Wall Street Journal database. Evaluation results show that the suggested scheme outperforms different conventional approaches in the case of short adaptation utterances. We also mathematically prove that the Gaussian means of the adapted model using the MLGR converges to their $M L$ estimates in the case of long adaptation utterances.
\end{abstract}

Index Terms - speaker adaptation, maximum likelihood, general regression neural networks, non-linear speaker adaptation

\section{INTRODUCTION}

In spoken human-machine interactions Speaker Adaptation (SA) methods are usually applied to adapt the machine to the speaking characteristics of a user. This adaptation to gender, age, dialect, acoustic environment, etc. improves the automatic speech recognition accuracy. In many commercial applications, Rapid Speaker Adaptation (RSA) is required [1]. For example, when ordering an item over the telephone or checking one's bank statement, RSA is required because the interaction may involve only a few dialog turns.

This work is supported by the European Commission as a Marie-Curie ITN-project (FP7-PEOPLE-ITN-2008), namely Bayesian Biometrics for Forensics (BBfor2), under Grant Agreement number 238803.
Although different methods have been introduced for SA of Gaussian mixture models (GMM), it is still a very challenging task. Lack of adaptation data may make conventional SA approaches such as Maximum-aPosteriori (MAP) [2] or Maximum Likelihood Linear Regression (MLLR) [3] inapplicable [1].

In MAP, every Gaussian is adapted based on data that can be assumed to be generated by it and consequently, MAP needs a large amount of adaptation data to influence the model effectively. In MLLR (and its constrained and unconstrained variants), adaptation data is applied to estimate transformation matrices which map the means and covariances of the SI model to the means and covariances of the speaker adapted model. This linear affine model approximates the required non-linear mapping from the SI to the speaker adapted model, which limits the asymptotic performance as more adaptation data become available, even when working with multiple transforms, each valid in a subset of the acoustic space. Another limitation is that the elements of the aforementioned matrices are poorly estimated due to shortage of adaptation data in RSA. Consequently, we will have a poor mapping, which leads to an inaccurate adapted model [4]. Therefore, it is too risky to use conventional MLLR for RSA.

In [19] we have introduced a non-linear approach based on General Regression Neural Networks (GRNN) for RSA. Continuing our last research, in this paper, a novel fast and reliable speaker adaptation method, namely Maximum Likelihood General Regression (MLGR), is developed by modifying the previous scheme. The main advantage of the proposed approach over the last method is that, the Gaussian means of the adapted model using the MLGR converges to their ML estimates in the case of long adaptation utterances. The proposed method enjoys several other advantages over conventional speaker adaptation methods. First, in MLGR, unlike almost all other speaker adaptation methods, no optimization task or calculation of unknown parameters is needed. These characteristics make it very suitable for RSA since poor estimation of unknown parameters, which happens in many adaptation methods such as MLLR, is avoided 
completely. Second, MLGR uses a non-linear regression making it capable of capturing possible nonlinearities in the adaptation mapping. Finally, the computational complexity of MLGR is significantly lower than that of other non-linear speaker adaptation methods such as Maximum Penalized Likelihood Kernel Regression (MPLKR) [4] and Kernel Ridge Regression (KRR) [5].

\section{BACKGROUND}

Consider a Speaker Independent Hidden Markov Model (SI-HMM) with the following state probability density function $(p d f)$.

$$
f\left(o_{t}\right)=\sum_{i=1}^{N} c_{i} \Delta\left(o_{t}, \mu_{i}, \Sigma_{i}\right)
$$

where $c_{i}$ is the mixture coefficient for the $i^{\text {th }}$ mixture component, $\Delta$ is a Gaussian probability density function with mean $\mu_{i}$ and covariance matrix $\sum_{i}$ and $N$ is the total number of Gaussians in the mixture.

\subsection{Conventional speaker adaptation methods}

Maximum Likelihood Re-estimation (MLRE) estimates the optimal value of the HMM Gaussian means to maximize the likelihood of the adaptation data [4]. To find the optimal value of the Gaussian means, the Expectation Maximization (EM) algorithm [18] is typically employed so that in each of its iterations, the value of the $i^{\text {th }}$ transformed mean, which is denoted as $\mu_{i}$, is calculated as follows.

$$
\begin{aligned}
& \mu_{i}^{*}= \\
& \underset{\mu_{i}}{\arg \max }\left[-\sum_{i=1}^{N} \sum_{t=1}^{T} \gamma_{i}(t)\left(o_{t}-\mu_{i}\right)^{\prime} \Sigma_{i}^{-1}\left(o_{t}-\mu_{i}\right)\right]
\end{aligned}
$$

where $\gamma_{i}(t)$ is the posterior probability (computed with the HMM parameters of the previous iteration) of the $i^{\text {th }}$ mixture component at time $t$, and $T$ is the number of adaptation frames.

$\mu_{i}$ " can be easily obtained using the following relation.

$$
\mu_{i}^{*}=\frac{\sum_{t=1}^{T} \gamma_{i}(t) o_{t}}{\sum_{t=1}^{T} \gamma_{i}(t)}
$$

If $\gamma_{i}(t)$ is too small, i.e. the phonetic context modeled by the Gaussian is not or hardly observed in the adaptation data, the update by (3) is inaccurate. In RSA this is often the case and only $M$ out of all $N$ Gaussians can be updated. Consequently, MLRE does not lead to an accurate adapted model in RSA.

\subsection{Transformation-based methods}

From the above discussion, we see that adaptation data should be used in more effective ways to reach more accurate adapted models. Progress can be made by assuming a transformation that maps the SI-HMM Gaussian parameters to their speaker-adapted equivalents. The parameters of the transformation are estimated on the observed Gaussians and subsequently applied to all the Gaussians, including the unobserved ones. While this overcomes a drawback of MLRE in RSA, it calls upon our imagination to produce a parameterized expression of the transformation that describes the mapping with sufficient accuracy. However, it is not a trivial task due to the high dimensionality of the space in which this transformation operates. Of course, any (sufficiently smooth) non-linear mapping can be approximated by a first order Taylor series, i.e. a linear mapping. Transformation-based methods are thus divided into linear transformation methods and non-linear schemes.

\subsubsection{Linear Transformation methods}

There are a number of methods which try to approximate a linear transformation to adapt the SI-HMM to the speech data of a new speaker. The flagship of these methods is MLLR [17]. The basic assumption of MLLR is that Gaussian mean parameters are updated according to following linear transformation.

$$
\begin{aligned}
& \hat{\mu}_{i}=W \xi_{i} \\
& \xi_{i}=\left[\begin{array}{ll}
\mu_{i}^{\prime} & 1
\end{array}\right]
\end{aligned}
$$

where $W$ is a matrix of proper size, and $\hat{u}_{i}$ is the new adapted mean of $i^{\text {th }}$ mixture component.

The transformation matrix $W$ is estimated based on the ML criterion such that the likelihood of adaptation data is maximized. There is a closed form solution for estimating $W$ using the EM algorithm. However, due to shortage of adaptation data in RSA, the elements of $W$ might still be poorly estimated. Consequently, we will have a poor mapping, which leads to an inaccurate adapted model [4]. Therefore, it is too risky to use conventional MLLR for RSA.

\subsubsection{Non-linear Transformation methods}

Non-linear transformation based methods such as KRR [5] and MPLKR [4] have been recently introduced to overcome different drawbacks of linear transformation approaches. The basic assumption of these methods is that Gaussian means are updated using a non-linear transformation $F$.

$\hat{\mu}_{i}=F\left(\mu_{i}\right)$

where $F: \mathbb{R}^{D} \rightarrow \mathbb{R}^{D}$ is a non-linear function of $\mu_{i}$. The method of approximating this non-linear transformation significantly influences the accuracy of adapted model. In approximation of $F$, usually, the values of $\mu_{i}$ " are used. However, this method cannot be used in many practical applications due to its large computational complexity. 


\section{PROPOSED METHOD}

To propose a novel non-linear transformation based method, we keep the assumption of relation (5). We also assume

$\hat{\mu}_{i}=F\left(\mu_{i}\right)=G\left(\mu_{i}\right)+\mu_{i} \quad 1 \leq i \leq N$

where $G: \mathbb{R}^{D} \rightarrow \mathbb{R}^{D}$ is a non-linear function of $\mu_{i}$. The idea of MLGR is to approximate the mapping $G$ using GRNN with the ML estimates as targets.

\subsection{Training dataset}

Based on the ML criterion, as discussed in section 2, the best values for the means of the observed Gaussians can be calculated using relation 3 . Therefore, the values of $\mu_{i}$ can be used to approximate $G$. Consequently, our training dataset is $S=\left\{\left(\mu_{1}, \mu_{1}^{*}-\mu_{1}\right) \cdots\left(\mu_{m}, \mu_{m}^{*}-\mu_{m}\right) \quad \cdots\right.$ $\left.\left(\mu_{M}, \mu_{M}^{*}-\mu_{M}\right)\right\}$.

Note that $M$ is an integer between 1 and $N$, which represents the number of observed Gaussians, i.e. those $i$ in (3) for which the denominator exceeds a threshold.

\subsection{Function approximation}

Our final goal is to approximate $G$ such that it learns the training examples and generalizes well on new data points. There are different ways to reach this goal such as different classical linear and non-linear regression methods [9] or different soft computing approaches [10]. Among those, however, we have chosen GRNN due to different characteristics making it suitable for this problem. Advantages of GRNN over other function approximation methods will be discussed later in this paper. GRNN, which is a universal approximator, was introduced by Donald Specht [11]. It is an approach with a one-pass learning algorithm. In GRNN, $G\left(\mu_{i}\right)$ is approximated as:

$\hat{G}\left(\mu_{i}\right)=\frac{\sum_{m=1}^{M} y_{m} \exp \left(-\frac{\left(\mu_{i}-\mu_{m}\right)^{\prime} \cdot\left(\mu_{i}-\mu_{m}\right)}{2 \sigma_{m}(T)^{2}}\right)}{\sum_{m=1}^{M} \exp \left(-\frac{\left(\mu_{i}-\mu_{m}\right)^{\prime} \cdot\left(\mu_{i}-\mu_{m}\right)}{2 \sigma_{m}(T)^{2}}\right)}$

$y_{m}=\left(\mu_{m}^{*}-\mu_{m}\right)$

where $\sigma_{m}(T)$ is the width of the $m^{\text {th }}$ radial basis function and $\mu_{m}$ is the $m^{\text {th }}$ input training vector. The procedure of determining $\sigma_{m}(T)$ is explained in section 3.3. Consequently, the approximated $F\left(\mu_{i}\right)$ in MLGR is

$\hat{\mu}_{i}=\hat{F}\left(\mu_{i}\right)=\hat{G}\left(\mu_{i}\right)+\mu_{i}$

Equation (8) can also be interpreted as a smoothing operation with extent $\sigma_{m}(T)$ of the observed mean corrections $y_{m}$ involved in adaptation.

\subsection{Determining hyperparameters}

The value of $\sigma_{m}(T)$, which is also called smoothing parameter, significantly impacts the function approximation accuracy [13-15]. In this research, a two stage procedure is proposed to determine the smoothing parameter. In the initialization stage, a common initial value based on the variance of the input data is determined for the standard deviation of all Gaussian functions. In the second stage, namely the modification stage, the initial value of the Gaussian standard deviation is modified for each Gaussian based on the amount of available adaptation data.

These stages are elaborated in more detail as follows.

\section{A. Initialization Stage}

It is shown in [13] that there exists a close relationship between the variance of the input data and the smoothing parameter. Therefore, the following choice is adopted in this paper.

$$
\begin{gathered}
\sigma_{\text {int }}^{2}=\alpha\|\operatorname{var}(U)\|_{2} \\
U=\left[\mu_{1}, \ldots, \mu_{N}\right]
\end{gathered}
$$

where $\operatorname{var}(U)$ is a column vector containing the variance of each row of $U$ and $\alpha$ is a constant that is determined on an independent development set. The procedure of obtaining $\alpha$ in our experiments is explained in section 4.2.

\section{B. Modification Stage}

In this stage, the smoothing parameter is modified for each Gaussian using the following relation.

$$
\sigma_{m}(T)^{2}=\frac{\tau \sigma_{\mathrm{int}}^{2}}{\tau+\sum_{t=1}^{T} \gamma_{m}(t)}
$$

where $\tau$ is a hyperparameter, usually between 100 and 1000 , which is determined offline. Gaussians with limited amounts of adaptation data will have a small accumulated posterior probability, such that their smoothing variance will approximate the initial value. The smoothing parameter is inversely proportional to the amount of training data for large $T$.

\subsection{Advantages of MLGR over other SA methods}

Many adaptation schemes such as kernel eigenvoice [6-7] and kernel eigenspace-based MLLR [8] involve nonconvex gradient-based optimization methods. An evident disadvantage pertaining to all gradient based optimization techniques is the occurrence of local extrema. In contrast, MLGR involves no optimization task or calculation of unknown parameters.

Another important advantage of MLGR is its computational simplicity. Table 1 shows the computational complexity of MLGR along with two other non-linear adaptation methods published recently. As can be interpreted from the table 1, the computational 
complexity of MLGR is significantly lower than that of MPLKR or KRR. This characteristic makes the MLGR suitable for real time applications.

Finally, for large amounts of adaptation data, the limiting behavior of MLGR is still that of MAP.

Table 1. Computational Complexity of KRR [5], MPLKR [4], and MLGR. $D$ is the vector dimension, $N$ is the number of Gaussians, $M$ is the number of observed Gaussians and $T$ is the

\begin{tabular}{|c|c|}
\multicolumn{2}{c}{ number of adaptation frames } \\
\hline Method & Computational Complexity \\
\hline KRR & $O\left(D N^{3} T^{3}\right)$ \\
\hline MPLKR & $O\left(N^{3}\right)$ \\
\hline MLGR & $O(D M N)$ \\
\hline
\end{tabular}

Theorem:

As the amounts of adaptation data increases toward infinity ( $T$ tends to infinity), MLGR estimate converges to ML estimate.

Proof:

From relation (14) we have

$\lim _{T \rightarrow \infty} \sigma_{m}(T)=0$

Hence

$\lim _{T \rightarrow \infty} F\left(\mu_{i}\right) \equiv \lim _{\sigma_{m} \rightarrow 0} F\left(\mu_{i}\right)$

On the other hand

$\lim _{\sigma_{m} \rightarrow 0} \exp \left(-\frac{\left(\mu_{i}-\mu_{m}\right)^{\prime} \cdot\left(\mu_{i}-\mu_{m}\right)}{2 \sigma_{m}(T)^{2}}\right)$

$= \begin{cases}1 & \text { if } i=m \\ 0 & \text { if } i \neq m\end{cases}$

Hence,

$$
\lim _{\sigma_{m} \rightarrow 0} \frac{\sum_{m=1}^{M} y_{m} \exp \left(-\frac{\left(\mu_{i}-\mu_{m}\right)^{\prime} \cdot\left(\mu_{i}-\mu_{m}\right)}{2 \sigma_{m}(T)^{2}}\right)}{\sum_{m=1}^{M} \exp \left(-\frac{\left(\mu_{i}-\mu_{m}\right)^{\prime} \cdot\left(\mu_{i}-\mu_{m}\right)}{2 \sigma_{m}(T)^{2}}\right)}=y_{i}
$$

Note that in the case of long adaptation data all HMM Gaussians are observed. Therefore, in this case $M$ is equal to $N$ and condition $i=m$ is satisfied for all inputs.

From relations (8), (12) and (14),

$$
\lim _{T \rightarrow \infty} F\left(\mu_{i}\right)=y_{i}-\mu_{i}=\mu_{i}^{*}=\frac{\sum_{t=1}^{T} \gamma_{i}(t) o_{t}}{\sum_{t=1}^{T} \gamma_{i}(t)}
$$

Therefore, MLGR is asymptotically optimal in the maximum likelihood sense.

\subsection{Advantages of GRNN over other NNs}

Different advantages of GRNN over other NNs, which are pointed out in [11-12], [15], are:

- GRNN does not require iterative learning algorithms. Instead, it has a one pass and fast learning. The standard supervised neural network architectures such as the Multilayer Perceptron (MLP) and RBF infer a parameterized model (the weights forming the parameters) from available training data. These networks use the back-propagation algorithm for training, where it may take a large number of iterations to converge to the desired solution.

- GRNN requires only a fraction of the training samples a back propagation based neural network would need. In other words, GRNN can be effectively applied in the case of very sparse data.

\section{EXPERIMENTAL RESULTS}

In this section, the efficacy of proposed method in comparison with three conventional methods, namely full transform MLLR (F-MLLR), diagonal transform MLLR (D-MLLR) and Maximum Penalized Likelihood Linear Regression (MPLLR) [4], is shown.

\subsection{Experiment setup}

The proposed adaptation method was investigated on the WSJ benchmark large vocabulary recognition task: $5 \mathrm{k}$ word closed vocabulary using the standard bigram language model on the November 92 evaluation set.

The feature extraction is MEL-spectrum based, including mean subtraction on the log spectrum, and a linear discriminant analysis resulting in 39 features. The baseline model doesn't incorporate Vocal Tract Length Normalization (VTLN).

Training of the acoustic models is based on the standard SI- 84 WSJ0 database which contains about 15 hours of speech in total from 84 speakers. Speaker independent, cross word context and position dependent acoustic models were generated with 1938 tied HMM states, defined by an automatic phonetic decision tree for 45 phones. In total 32064 Gaussians with diagonal covariance were estimated. There is no sharing of Gaussians across states.

\subsection{Tuning the hyperparameters}

The optimal value for $\alpha$ for MLGR is found by a linear search using a word error rate (WER) criterion on the November 92 development set. Each of the 10 speakers in this set has about 40 utterances. 30 of these were used for testing and of the remaining utterances, those with a maximum length of 10 seconds were used for adaptation. The smoothing variance was set according to its initial value (11). Table 2 shows the average WER over all adaptation experiments of all speakers for different values of $\alpha$. 
As can be interpreted from table 2, the lowest average WER is obtained where $\alpha$ is equal to 0.5 . Therefore, we adopt this value in rest of our experiments.

Table 2. Average WER in $\%$ for different values of $\alpha$

\begin{tabular}{|c|c|c|c|c|c|}
\hline$\alpha$ & $1 / 8$ & $2 / 8$ & $4 / 8$ & $6 / 8$ & $8 / 8$ \\
\hline $\begin{array}{c}\text { Average } \\
\text { WER }\end{array}$ & 26.54 & 11.53 & $\mathbf{7 . 9 7}$ & 8.29 & 8.41 \\
\hline
\end{tabular}

\subsection{Results}

To evaluate the proposed method two experiments have been performed.

The November 92 evaluation data set includes utterances of 8 different speakers. Very short utterances, which are available only for speakers 1, 2, 3 and 4, are used to assess MLGR in very rapid speaker adaptation cases. Speakers 5, 6, 7 and 8, which have longer utterances, are used to evaluate the proposed method when the available adaptation data is between 5 seconds to 10 seconds.

Experiment 1:

In this experiment, our aim is to assess MLGR when the duration of the adaptation speech is less than 5 seconds. The initial SI model is adapted to four different speakers. For each speaker, the SI model is adapted using four different utterances (Utt) with maximum length of 5 seconds. After the training phase, each adapted model is tested on about thirty sentences of the evaluation data.

Table 3 indicates the Word Error Rate (WER) obtained using the SI model and the adapted model applying F-MLLR, D-MLLR, MPLLR, and MLGR for four different speakers. The duration of the adaptation utterances (in seconds) is also listed for each test. Note that for each method 10 adaptation iterations were run.

As it can be interpreted from table 3, MLGR is the most accurate method in rapid speaker adaptation in comparison with the other three methods.

Analysis of these results show, in average, relative word error rate reduction compared to SI, F-MLLR, DMLLR and MPLLR is $24.5 \%, 70.7 \%, 10.9 \%, 8 \%$ respectively. This experiment also shows that F-MLLR can fail due to poor estimation of the transformation matrix when the adaptation data is very scarce.

Experiment 2:

In this experiment, our aim is to assess MLGR when the duration of adaptation speech is a little longer than the first experiment (between 5 seconds to 10 seconds). Therefore, all adaptation utterances of this experiment are in this interval. The initial SI model is adapted to four new speakers (other than speakers in the first experiment).

For each speaker, the SI model is adapted using an adaptation utterance. After the training phase, each adapted model is tested on thirty utterances. To ensure the evaluation process, the training and testing phases are repeated five times for each speaker using seven different adaptation utterances. Table 4 illustrates the Average
WER (AWER) for each speaker. Note that for each method 10 adaptation iterations were run. Again, MLGR outperforms the other methods. Table 4 suggests MLGR achieves relative word error rate reduction of $21 \%, 23 \%$, $6 \%$ and $11 \%$ over SI, F-MLLR, D-MLLR and MPLLR respectively.

Table 3. Performance of F-MLLR, D-MLLR, MPLLR and MLGR in the first experiment. Results are WER in \%

\begin{tabular}{|c|c|c|c|c|c|c|}
\hline & Utt 1 & Utt 3 & Utt 3 & Utt 4 & Average \\
\hline \multirow{6}{*}{$\begin{array}{l}\overline{\vec{s}} \\
\bar{s} \\
\text { के }\end{array}$} & Utt Length (sec) & 4.45 & 4.6 & 4.67 & 4.71 & 4.61 \\
\hline & SI & 10.2 & 10.2 & 10.2 & 10.2 & 10.2 \\
\hline & F-MLLR & 16.3 & 15.1 & 16.7 & 13.4 & 15.37 \\
\hline & D-MLLR & 8.25 & 8.64 & 7.47 & 6.88 & 7.81 \\
\hline & MPLLR & 8.45 & 8.64 & 9.04 & 8.45 & 8.64 \\
\hline & MLGR & 7.47 & 6.48 & 6.68 & 6.88 & 6.88 \\
\hline \multirow{6}{*}{$\begin{array}{l}N \\
\bar{d} \\
\text { s. } \\
\text { है } \\
\text { है }\end{array}$} & Utt Length (sec) & 4.1 & 4.11 & 4.16 & 4.96 & 4.3 \\
\hline & SI & 4.7 & 4.7 & 4.7 & 4.7 & 4.7 \\
\hline & F-MLLR & 22.9 & 28.2 & 15.8 & 9.62 & 19.13 \\
\hline & D-MLLR & 5.98 & 6.2 & 6.62 & 5.34 & 6.03 \\
\hline & MPLLR & 4.06 & 4.27 & 4.27 & 4.27 & 4.22 \\
\hline & MLGR & 3.63 & 4.06 & 3.85 & 4.49 & 4.01 \\
\hline \multirow{6}{*}{ 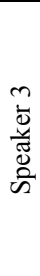 } & Utt Length (sec) & 4.35 & 4.4 & 4.51 & 4.55 & 4.45 \\
\hline & SI & 4.89 & 4.89 & 4.89 & 4.89 & 4.89 \\
\hline & F-MLLR & 8.51 & 11.2 & 15.2 & 8.88 & 10.95 \\
\hline & D-MLLR & 4.53 & 4.53 & 4.71 & 5.25 & 4.75 \\
\hline & MPLLR & 4.71 & 4.89 & 4.71 & 4.71 & 4.75 \\
\hline & MLGR & 4.71 & 4.71 & 4.17 & 4.17 & 4.44 \\
\hline \multirow{6}{*}{$\begin{array}{l}+ \\
\vec{d} \\
\text { ज्ञ } \\
\text { है } \\
\text { की }\end{array}$} & Utt Length (sec) & 3.91 & 3.95 & 4.63 & 4.75 & 4.31 \\
\hline & SI & 11.1 & 11.1 & 11.1 & 11.1 & 11.1 \\
\hline & F-MLLR & 40.8 & 57.3 & 18.4 & 19.6 & 34.02 \\
\hline & D-MLLR & 7.18 & 7.77 & 7.57 & 7.96 & 7.62 \\
\hline & MPLLR & 7.77 & 7.77 & 7.77 & 7.77 & 7.77 \\
\hline & MLGR & 7.96 & 6.99 & 8.35 & 8.74 & 8.01 \\
\hline
\end{tabular}

Table 4. Performance of F-MLLR, D-MLLR, MPLLR and MLGR in the second experiment. Results are AWER

\begin{tabular}{|c|c|c|c|c|c|}
\multicolumn{5}{|c|}{ in $\%$} & \\
\cline { 1 - 5 } Method $\rightarrow$ & SI & F-MLLR & D-MLLR & MPLLR & MLGR \\
\cline { 1 - 5 } $\begin{array}{c}\text { Speaker } \\
\text { Number } \\
\downarrow\end{array}$ & & & & & \\
\hline $\mathbf{5}$ & 13.98 & 21.22 & 11.13 & 12.12 & 10.35 \\
\hline $\mathbf{6}$ & 11.23 & 10.87 & 10.28 & 11.12 & 10.46 \\
\hline $\mathbf{7}$ & 8.25 & 6.13 & 6.32 & 7.53 & 6.29 \\
\hline $\mathbf{8}$ & 10.86 & 7.16 & 9.27 & 8.61 & 7.84 \\
\hline Average & 11.08 & 11.34 & 9.25 & 9.84 & $\mathbf{8 . 7 3}$ \\
\hline
\end{tabular}

\section{CONCLUSIONS}

In this paper, a novel non-linear approach for speaker adaptation was introduced and evaluated on an RSA task. 
In this method, which is called Maximum Likelihood General Regression or MLGR, a GRNN trained based on the ML criterion is applied to adapt the SI Gaussian means. The proposed adaptation approach enjoys four important advantages. First, since the MLGR does not involve estimating unknown parameters, this method can be applied even if the available adaptation data is very limited. Second, it can capture non-linearities in the adaptation mapping. Third, this method is very simple and computationally more efficient than many other nonlinear approaches such as MPLKR or KRR. Fourth, it converges to MAP adaptation with increasing adaptation data.

Evaluation results over WSJ benchmark confirm that the proposed scheme exhibits higher recognition accuracies in comparison with F-MLLR, D-MLLR and MPLLR.

\section{REFERENCES}

[1] R. Kuhn, F. Perronnin, and J. Junqua, "Time is money: Why very rapid adaptation matters," in proc. ISCA Workshop on Adaptation Methods for Speech Recognition, France, pp. 33-36, 2001.

[2] J. L. Gauvain and C. H. Lee, "Maximum a posteriori estimation for multivariate Gaussian mixture observation of Markov chains," IEEE Trans. Speech Audio Process., vol. 2, pp. 291-298, Apr. 1994

[3] C. J. Leggeter and P. C. Woodland, "Maximum Likelihood Linear Regression for Speaker Adaptation of Continuous Density Hidden Markov Models," Comput. Speech Lang., vol. 9, pp. 171-185, 1995.

[4] B. K. Mak, T. Lai, I. W. Tsang, and J. T. Kwok, "Maximum Penalized Likelihood Kernel Regression for Fast Adaptation," IEEE Trans. Audio, Speech, Lang. Process., vol. 17, no. 7, pp. 1372-1381, 2009.

[5] G. Saon, "A non-linear speaker adaptation technique using kernel ridge regression," in Proc. IEEE Int. Conf. Acoust., Speech, Signal Process., 2006, vol. I, pp. 225-228.

[6] B. Mak, J. T. Kwok, and S. Ho, "Kernel eigenvoice speaker adaptation," IEEE Trans. Speech Audio Process., vol. 13, no. 5, pp. 984-992, Sep. 2005.

[7] B. Mak, R. Hsiao, S. Ho, and J. T. Kwok, "Embedded kernel eigenvoice speaker adaptation and its implication to reference speaker weighting," IEEE Trans. Speech Audio Process., vol. 14, no. 4, pp. 1267-1280, 2006.

[8] B. Mak and R. Hsiao, "Kernel eigenspace-based MLLR adaptation," IEEE Trans. Audio, Speech, Lang. Process., vol. 15 , no. 3, pp. 784-795, 2007.

[9] D. A. Freedman, "Statistical Models: Theory and Practice," Cambridge University Press, 2005.

[10] R. A. Aliev and R. R. Aliev, "Soft computing and its applications," World Scientific, 2001.

[11] D. F. Specht, "A general regression neural network," IEEE Trans. Neural Networks, vol. 2, no. 6, pp. 568-576, 1991.
[12] C. Christodoulou and M. Georgiopoulos, "Application of Neural Network in Electromagnetics," Boston: Artech House, pp. 149-160, 2001.

[13] M. Zhong, et. al., "Gap-Based Estimation: Choosing the Smoothing Parameters for Probabilistic and General Regression Neural Networks" Int. Conf. Neural Networks, Canada, pp. 1870-1877, 2006.

[14] D. F. Specht, "Experience with Adaptive Probabilistic Neural Networks and Adaptive General Regression Neural Networks," in Proc. IEEE World Cong. Computational Intelligence, vol. 2, pp. 1203-1208, 1994.

[15] B. Erkmena and T. Yildırım, "Improving classification performance of sonar targets by applying general regression neural network with PCA" Expert Sys. Appl., vol. 35 , no. 1-2, pp. 472-475, 2008.

[16] J. Y. Goulermas, P. Liatsis, X. Zeng and P. Cook, "Density-Driven Generalized Regression Neural Networks for Function Approximation," IEEE Trans. Neural Networks, vol. 18, no. 6, pp. 1683-1696, 2007.

[17] M. J. F. Gales and P. C. Woodland, "Mean and Variance Adaptation within the MLLR Framework," Comput. Speech Lan., vol. 10, no. 4, pp. 249-264, 1996.

[18] V. Digalakis, D. Rtischev and L. Neumeyer, "Speaker Adaptation Using Constrained Reestimation of Gaussian Mixtures," IEEE Trans. Speech and Audio Processing, vol. 3, no. 5, pp. 357-366, 1995.

[19] M. H. Bahari and H. Van hamme, "Rapid speaker adaptation using maximum likelihood neural regression," in proc. IEEE Int. Conf. Multimedia and Expo, Spain, 2011. 